\title{
les surfaces de glissement en mécanique des sols
}

\author{
P. HABIB \\ Laboratoire de mécanique des solides \\ (Laboratoire commun E.P.-E.N.S.M.P.-E.N.P.C. - Associé au C.N.R.S.) \\ Ecole polytechnique, 91128 Palaiseau Cedex
}

\section{INTRODUCTION}

La localisation de la déformation quasí statique sur une ou plusieurs surfaces de glissement est un phénomène très courant en mécanique des sols ou en mécanique des roches (fig. 1). II n'a cependant pas fait l'objet de nombreuses publications et la théorie classique de la plasticité (Mandel, 1966) laisse de côté les déformations hétérogènes. C'est pourtant un sujet important, mais il est difficile car le phénomène physique de l'apparition de la localisation se prête mal à l'observation, le sol n'étant pas transparent. En fait, on ne connait bien les surfaces de glissement que par leur émergences et les praticiens savent que la recherche par sondages ou par tranchées d'une surface de glissement en profondeur, après un accident, est une opération coûteuse mais parfois décevante, les surfaces pariétales ayant une regrettable tendance à se recoller parfaitement. Quant à la progression des surfaces de glissement elles-mêmes, les observations sont parcellaires et ce n'est que très récemment que quelques données ont pu être recueillies sur la cinétique du développement des surfaces de glissement. Ainsi, Suemine (1983) a indiqué des vitesses de progression de la surface de glissement en profondeur de l'ordre de 1 à $100 \mathrm{~m} / \mathrm{h}$.

Mais, même pour une situation apparemment très élémentaire et très accessible, comme par exemple un essai de compression simple ou un essai triaxial, l'orientation de la ou des surfaces de glissement, par rapport à la direction des contraintes principales, reste imprécise et les comptes-rendus d'essais qu'on peut trouver sur ce sujet sont parfois contradictoires. Cette détermination présente en effet de sérieuses difficultés, d'une part parce que les lignes de glissement ne se matérialisent sur la surface latérale d'une éprouvette qu'après une certaine déformation, de l'ordre de 3 à $10 \%$ pour les sols, de sorte que les axes de références sont eux-mêmes déformés et on ne sait pas si l'on doit rattacher la surface de glissement à la configuration initiale ou à la configuration actuelle. Mais, d'autre part, il existe une certaine dispersion de l'orientation des lignes de glissement. Elle est particulièrement visible sur la figure 2 où l'on voit nettement, dans un champ qui fut homogène, deux familles conjuguées de surfaces de glissement dont l'une a manifestement été active postérieurement à l'autre, ce qui a entraîné l'apparition de rejets, mais aussi où certaines surfaces de la deuxième famille présentent un parallélisme douteux, à $15^{\circ}$ près. Sur la figure 3 , l'identification des deux familles conjuguées pose même des problèmes délicats. Cette imprécision de l'orientation se comprend aisément. Si l'on reprend le raisonnement de Coulomb, qui définit l'orientation du plan de rupture par le calcul du minimum strict d'une certaine résistance le long de ce plan en fonction de son angle d'inclinaison, il est bien évident que les plans d'inclinaisons voisines sont presque aussi défavorables que le plan critique. II suffit alors d'une légère hétérogénéité de la matière pour que le tracé de la surface de glissement devienne 


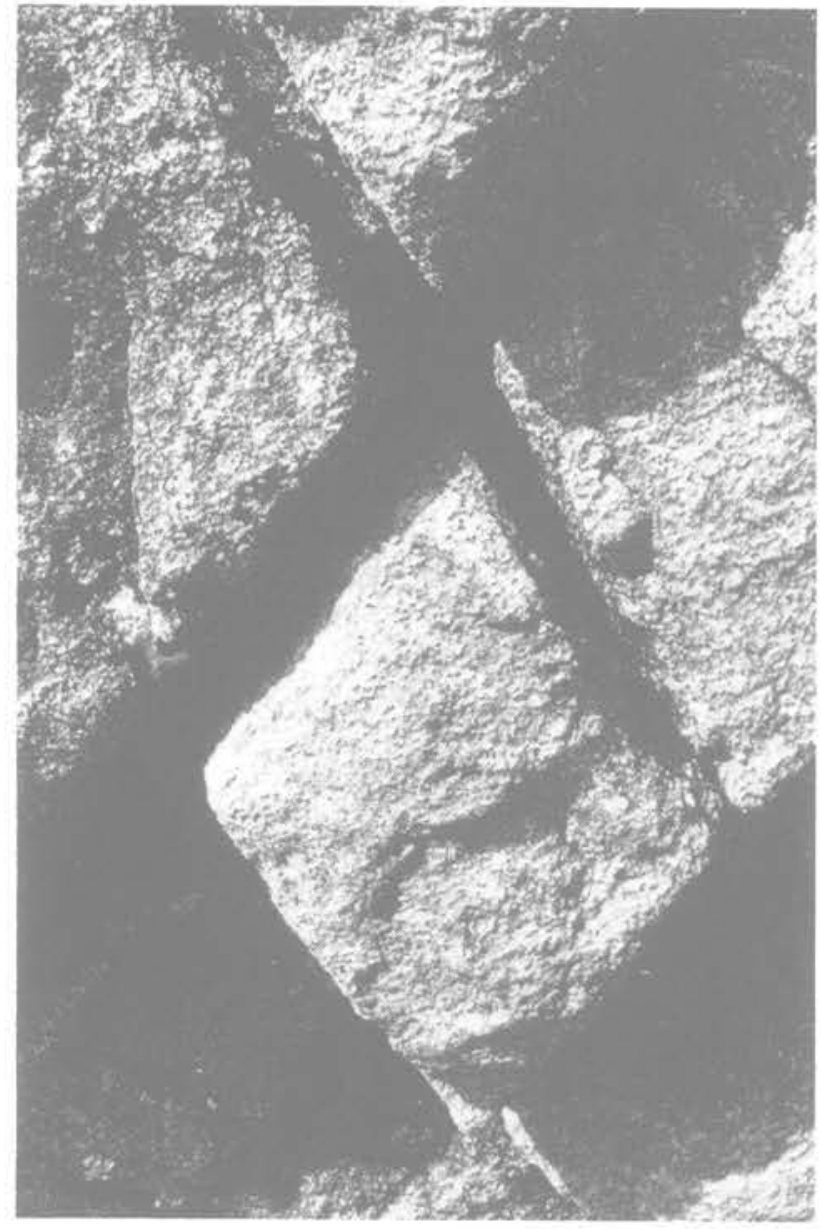

Fig. 1. - Lignes de glissement dans du granite. Les surfaces en contact ont été ouvertes par l'altération.

hésitant et, de fait, l'examen d'une surface de rupture montre qu'elle est constituée d'une série de petites facettes élémentaires dont la direction moyenne donne l'orientation générale.

Pourtant, les problèmes posés par les surfaces de glissement sont importants. Il est bien connu par exemple que les épicentres des séismes sont situés sur des failles actives, c'est-à-dire sur des surfaces de glissement.

A une échelle plus modeste, mais d'une façon tout aussi catastrophique, la figure 4 montre l'effet d'une surface de glissement sur un bâtiment. Cet accident spectaculaire est particulièrement remarquable, en ce sens que le déplacement de glissement y est de l'ordre du mètre, alors que l'émergence du glissement (fig. 5) s'est produite à environ $250 \mathrm{~m}$ de là: c'est dire que si la déformation avait été répartie au lieu d'être localisée, la maison n'aurait pratiquement pas subi de désordres.

Enfin, la nature même des surfaces de glissement est sujette à discussion. Dans les problèmes plans, les lignes de glissement sont-elles confondues avec les lignes caractéristiques de contraintes, comme on Pa cru

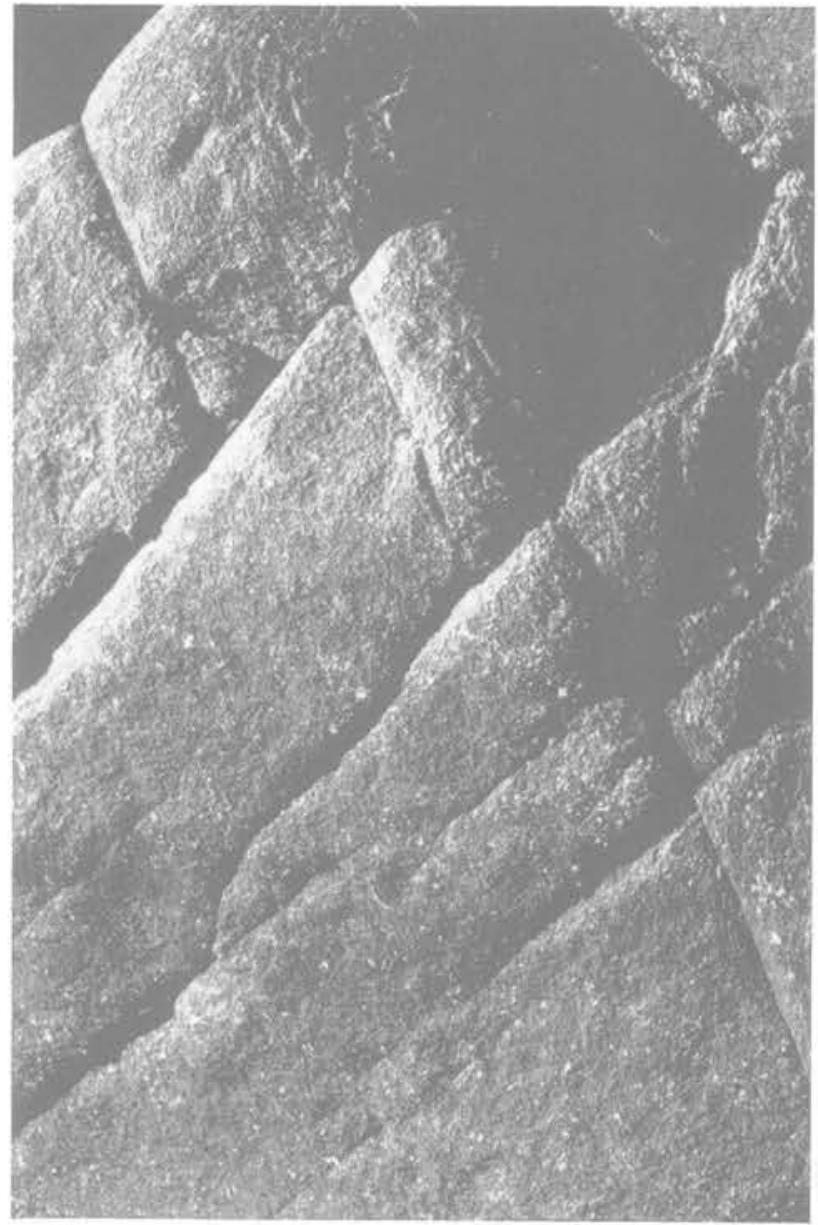

Fig. 2, - Deux familles de lignes de glissement avec rejet sur la première lors du jeu de la seconde. Noter le parallélisme incertain des orientations des lignes de la deuxième famille.

longtemps, ou avec les lignes caractéristiques des vitesses de déformation (lignes d'extension nulle), comme Roscoe (1970) l'a suggéré? L'étude de ce phénomène apparaît donc comme très difficile. On va voir cependant que son intérêt est grand et qu'il en découle des conséquences importantes.

Le premier sujet d'étonnement que l'on peut avoir vient du fait que dans certains cas, la rupture du sol se produit avec formation d'une surface de glissement mais qu'au contraire dans d'autres cas, le dépassement du seuil de résistance se produit avec une déformation tout à fait continue et sans localisation de la déformation. Par exemple, des surfaces de glissement apparaissent avec certains sols, comme les argiles raides ou les sables denses, mais pas avec d'autres, comme les matériaux compressibles, les vases ou les sables lâches. Parfois, les surfaces de glissement apparaissent sous contraintes moyennes modérées et disparaissent sous contraintes élevées; c'est le cas des sables moyennement denses et, dans un domaine voisin, celui des roches, où la transition fragile-ductile passe par un état intermédiaire où se forment des surfaces de glissement. Elles sont bien identifiées pour les roches et correspondent notamment aux failles et au chevauchement de la Géologie (Goguel, 1983). 


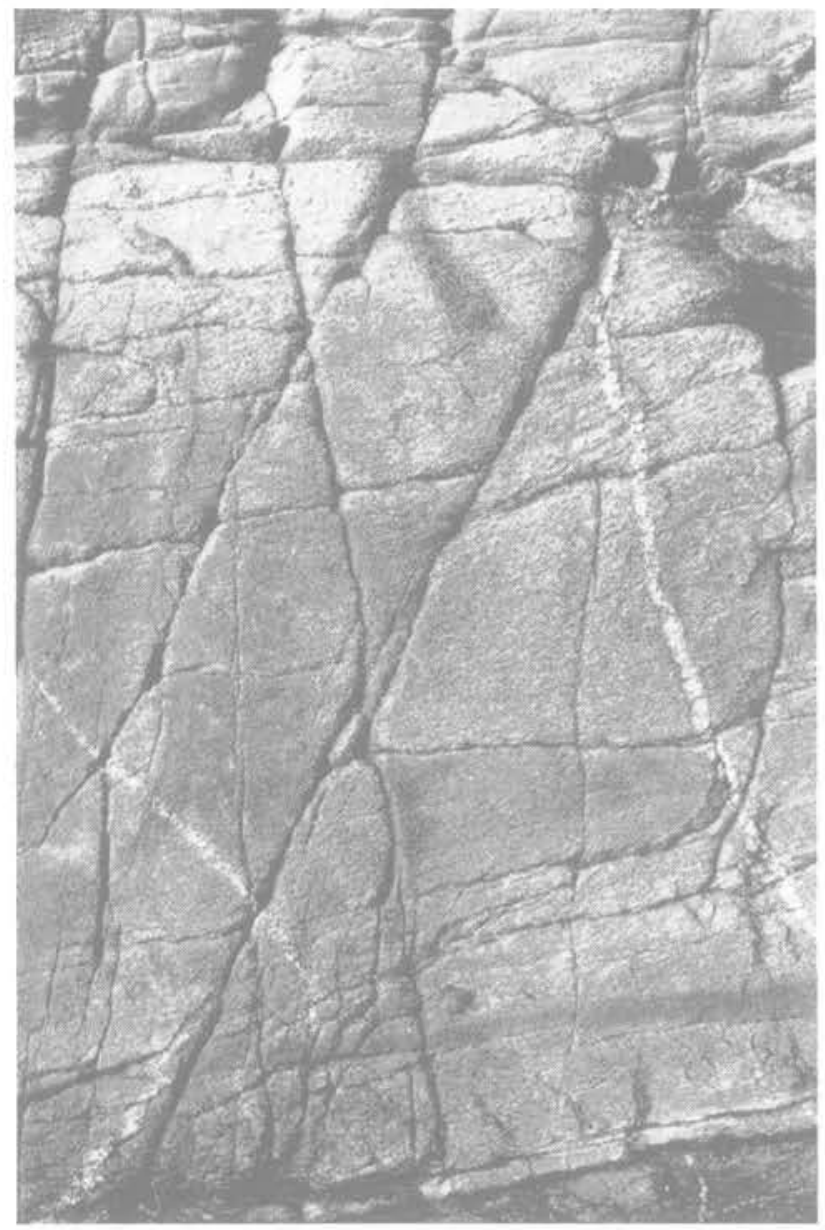

Fig. 3. - Dispersion des orientations des surfaces de glissement dans un massif de granite.

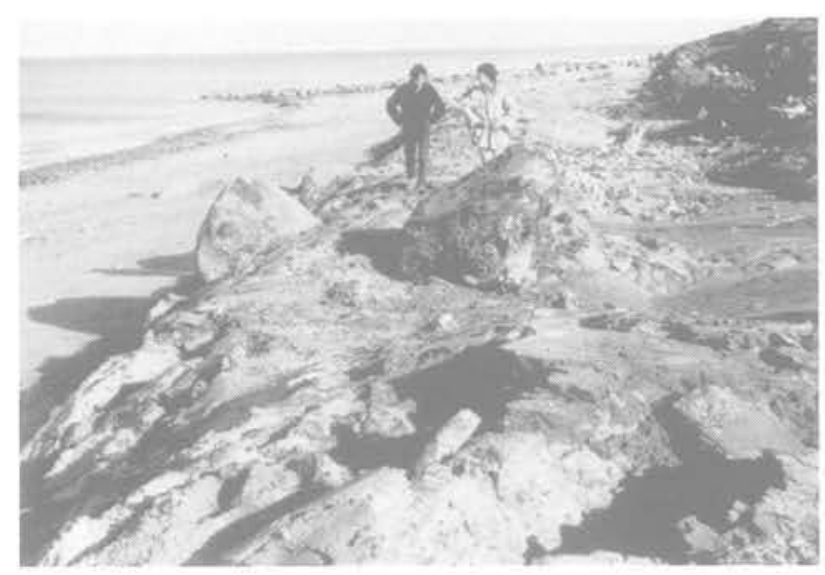

Fig. 5. - Glissement de terrain à Villerville. Emergence de la surface de glissement en son pied. (cliché B.R.G.M.).

Mais pour certains champs de contraintes, avec des matériaux où des surfaces de glissement se produisent habituellement, il y a des cas où les surfaces de ruptures apparaissent, comme par exemple les glissements de terrains, ou au contraire n'apparaissent pas, comme sous la pointe des pieux.

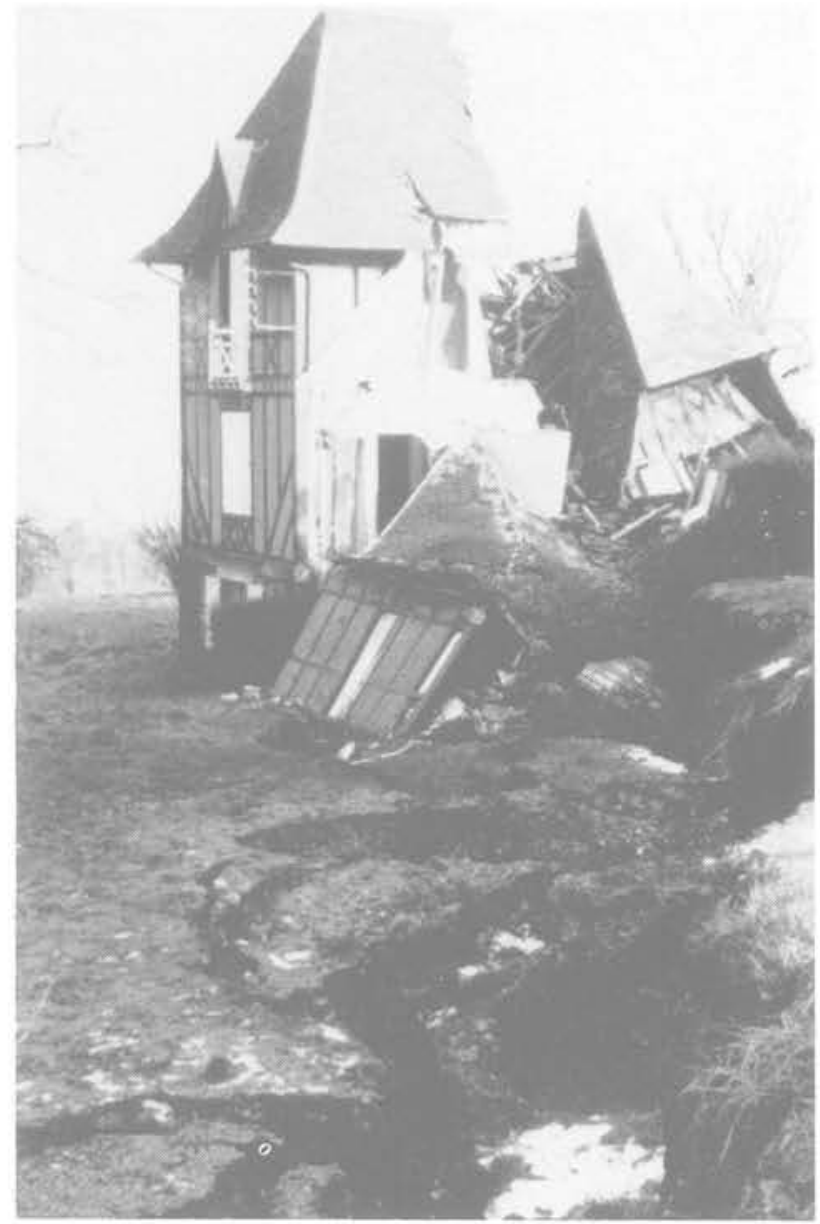

Fig. 4. - Glissement de terrain à Villerville. (cliché B.R.G.M.).

On voit donc que ce problème est très difficile en général puisque le critère de formation d'une surface de glissement doit tenir compte à la fois de la loi de comportement du matériau, de la contrainte moyenne, de la nature du champ de contraintes et probablement des conditions aux limites.

Nous allons examiner d'abord ce qui se passe dans un champ de contraintes homogène avant d'étudier le cas général.

\section{SURFACES DE GLISSEMENT EN CHAMP HOMOGËNE}

Dans un champ de contraintes homogène, lexpérience montre les phénomènes suivants: si la loi de comportement du matériau présente de l'écrouissage, la déformation est homogène; si la loi de comportement présente du radoucissement, il y a formation d'une surface de glissement. L'interprétation classique est la suivante. La matière réelle n'est jamais strictement homogène et il existe une petite dispersion des caractéristiques mécaniques représentée par le faisceau des courbes effort-déformation de la figure 6 , qui 

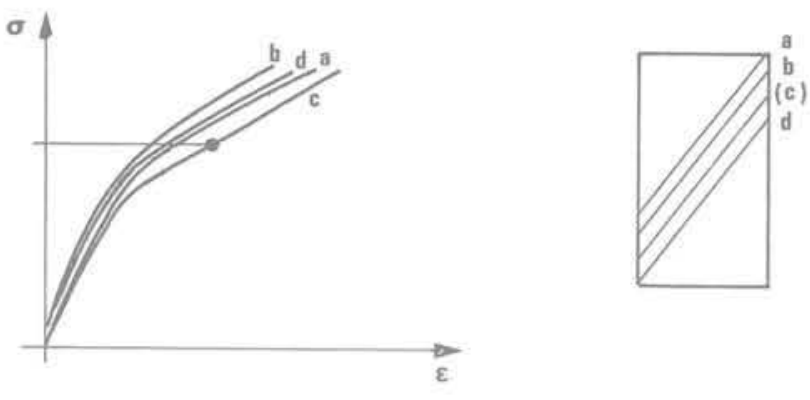

Fig. 6. - Cas de l'écrouissage.

indiquent les comportements d'une série de plans de glissement potentiels d'une éprouvette d'essai.

Soit (c) le plan le plus faible. Sous la contrainte $\sigma$, il subit un certain glissement. Un accroissement de la déformation autour de ce plan ne peut se produire que par un accroissement de la contrainte qui engendre à son tour l'augmentation de la déformation auprès des plans voísins et même si la déformation y est plus petite que dans le plan qui a cédé le premier, on voit que la déformation s'étend et se développe dans toute l'éprouvette.

Si au contraire, les courbes de comportement présentent un maximum (fig. 7), il existe un plan où la plus grande résistance est atteinte avant les autres.
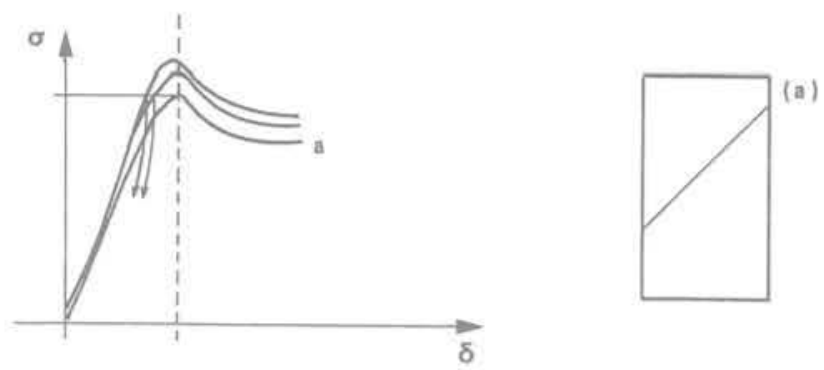

Fig. 7. - Cas du radoucissement.

Si la déformation augmente, la contrainte se met à décroître dans ce plan, ce qui provoque une détente dans toute l'éprouvette. Le module de Young d'un sol est beaucoup plus élevé au déchargement que le module de premier chargement. Il en résulte que les domaines qui n'ont pas été atteints par les grandes déformations se comportent désormais comme des blocs rigides qui coulissent sur le plan de glissement: il y a localisation de la déformation. On notera sur la figure 7 que le diagramme contrainte-déformation n'a plus grand sens après le maximum parce que la déformation n'est plus continue et il est nécessaire de le remplacer par un diagramme contraintedéplacement. Pour un essai triaxial sur du sable dense, on remarquera de la même façon que le diagramme de la variation relative de volume $\frac{\Delta V}{V}$ en fonction de la déformation (fig. 8) perd sa signification après le maximum de la résistance et qu'il doit être remplacé par la courbe de la variation de volume $\Delta \mathrm{V}$ en fonction du déplacement $\delta$,

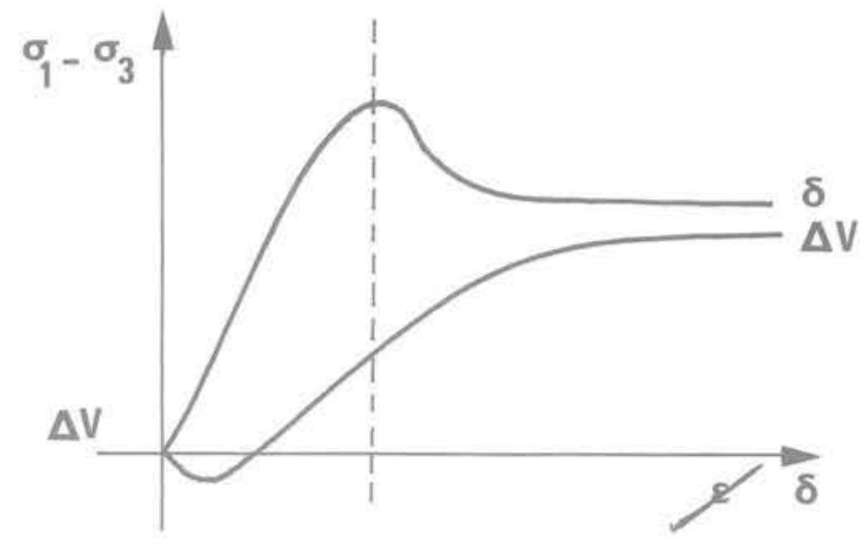

Fig. 8. - Variation de volume d'un sable dense en cours de rupture.

Cette remarque est importante car si la formation d'une surface de glissement est perceptible sur la courbe contrainte-déplacement, puisqu'elle apparaît et se développe physiquement une fois le maximum de la résistance dépassé, par contre elle n'est pas décelable sur la courbe des variations de volume. Il n'en reste pas moins que les variations de volume se sont localisées à un moment donné dans le plan de glissement, alors qu'elles ont cessé dans les blocs monolithes dès que la détente a commencé.

\section{SURFACES DE GLISSEMENT EN CHAMP NON HOMOGĖNE}

Examinons maintenant ce qui se passe dans un champ de contraintes non uniforme. Soit un solide soumis à des sollicitations quelconques (fig. 9). Lorsqu'elles aug-

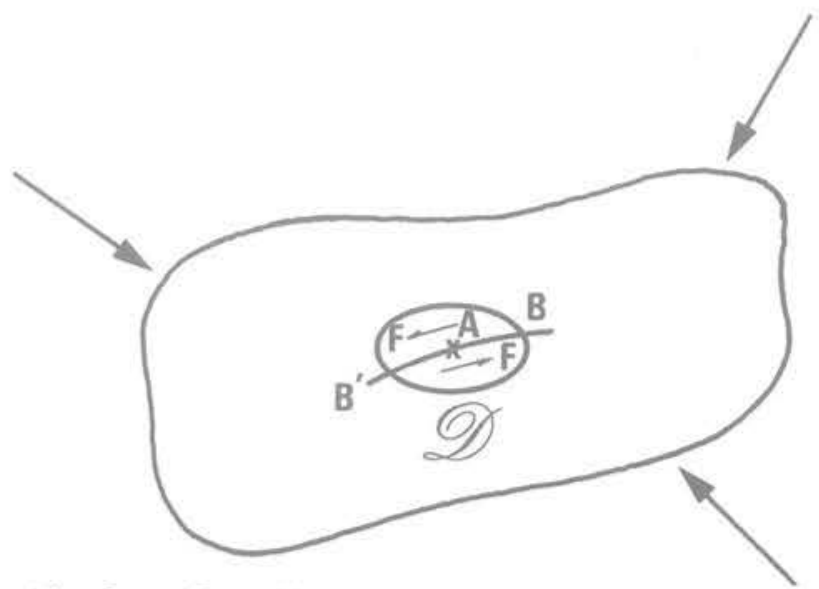

Fig. 9. - Franchissement du critère de plasticité. 
mentent, le critère de rupture est d'abord atteint en un point $A$ puis dans un petit domaine $D$ entourant le point $A$.

Si ce domaine est suffisamment petit, le champ de contraintes peut y être considéré comme uniforme et il y aura formation de surfaces de glissement si le corps est radoucissant ou déformation continue s'il est écrouissable. Plaçons-nous dans le cas de la mécanique des sols avec un radoucissement suffisamment petit pour que la solution élasto-plastique classique puisse être considérée comme une bonne approximation du problème étudié (*).

Regardons ce qui se passe sur la surface de glissement $A B$ de la figure 9 et devant elle dans son prolongement (fig. 10). A une certaine distance, la droite (a) perpendiculaire à la fissure n'est pratiquement pas déformée. La déformation est plus grande pour la droite (b) lorsqu'on s'approche de $\mathrm{B}$. Elle est maximale pour la droite (c) qui passe par B. Entre A et B, il se produit un glissement $\delta$ entre les lèvres de la fissure qui coupe les droites (d) et (e). Le glissement $\delta$ augmente de $B$ vers $A$. On a porté le déplacement $\delta(\mathrm{s})$ et le cisaillement correspondant $t(\mathrm{~s})$ sur la figure 10 (en supposant $\varphi=0$ pour simplifier l'exposé, mais on généraliserait facilement pour le cas $\varphi \neq 0$ ).

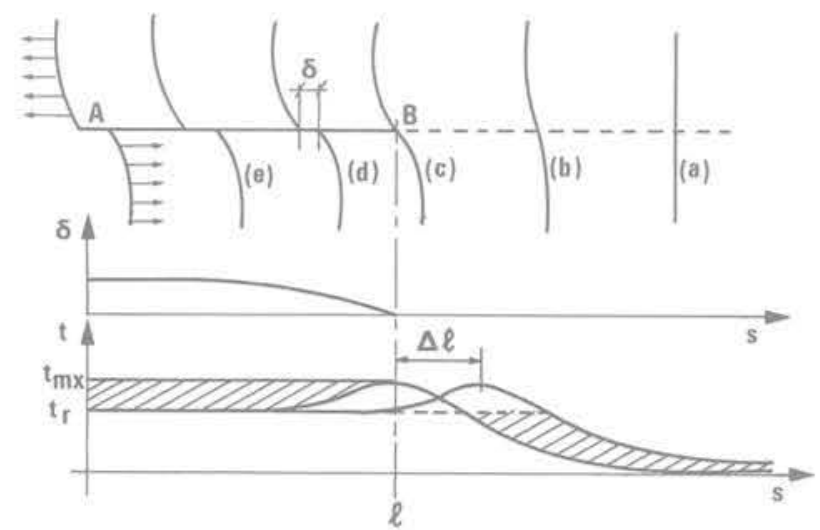

Fig. 10. - Déplacement et contrainte de cisaillement au voisinage d'une surface de glissement.

Dans une solution élasto-plastique classique, le cisaillement serait constant le long de $A B$ et il lui correspondrait un effort de cisaillement $F$ dans chaque moitié du domaine $\mathscr{D}$. Dans le cas du radoucissement, il existe dans le plan de la fissure $A B$, un déficit de cisaillement marqué en hachure sur la figure 10 , et dont la valeur totale:

$$
\Delta F=\int_{A B}\left(t_{m x}-t(s)\right) d s
$$

est majorée par $\left(t_{m x}-t_{r}\right) \ell$, où $t_{m x}$ est la plus grande résistance, $t_{\mathrm{r}}$ est la résistance au cisaillement résiduelle et $\ell$ la longueur du segment $A B$. L'équilibre ne peut être assuré que par un déplacement vers la droite en mode II de l'extrémité de la fissure, avec perturbation du champ élastique au-delà, de façon telle qu'avec les approximations de la figure 10 , les aires hachurées soient égales. Le déplacement $\Delta \ell$ de l'extrémité de la fissure permet de compenser le déficit de cisaillement $\triangle F$ en apportant une résistance supplémentaire $\Delta \ell . t_{r} ;$ le déplacement $\Delta \ell$ est donc majoré par:

$$
\Delta \ell<\left(\frac{t_{m x}}{t_{\mathrm{r}}}-1\right) \ell
$$

ou encore:

$$
\frac{\Delta \ell}{\ell}<\frac{t_{m x}}{t_{r}}-1 \text {. }
$$

Ainsi, si le radoucissement correspond à une résistance résiduelle de $0,9 t_{\mathrm{mx}}$, l'allongement de la fissure qui permet de rétablir l'équilibre est de $10 \%$. Ce mécanisme n'est possible que si le ligament est capable de supporter l'augmentation de la contrainte élastique, c'est-â-dire si l'aire de la partie ponctuée de la figure 10 bis est au moins égale à l'aire hachurée.

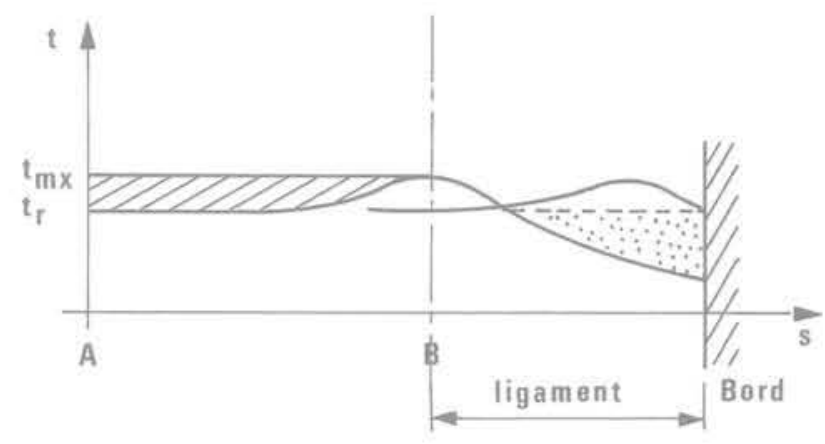

Fig. 10 bis. - Résistance ultime du ligament.

Dans le cas contraire, la plastification est totale, la surface de glissement atteint le bord libre et la rupture se produit. Mais, si la déformation plastique reste contenue, le mécanisme précédent bloque le mouvement de la surface de glissement en permettant à la déformation de se concentrer sur une autre surface de glissement, peut-être pas au voisinage immédiat de la première car on concevrait mal qu'il soit possible d'y supporter $t_{m x}$ alors que la première ne peut plus supporter que $t_{r}$; on peut donc prévoir l'apparition d'une multitude de surfaces de glissement discrètes et côte à côte, difficilement visibles parce que situées à l'intérieur du massif plastifié mais qui permettent de revenir au concept de déformation quasi homogène. Ceci est conforme à l'expérience.

La figure 11 représente une multitude de lignes de Luders discrètes à la surface d'une tôle emboutie, qui est une bonne image de la plastification autour d'un tunnel de section circulaire. Les lignes de Luders ne sont pas des lignes de glissement mais des lignes de grand allongement. A l'inverse des lignes de glissement qui apparaissent après une déformation plastique importante et se forment par un processus de condensation, les lignes de Luders qui se produisent dans certains métaux, comme l'aluminium ou l'acier doux, apparaissent immédiatement après le dépassement de la limite d'élasticité puis s'élargissent progressivement

(*) Ce ne serait généralement pas vrai en mécanique des roches où l'effondrement de la résistance après le maximum est très important. 


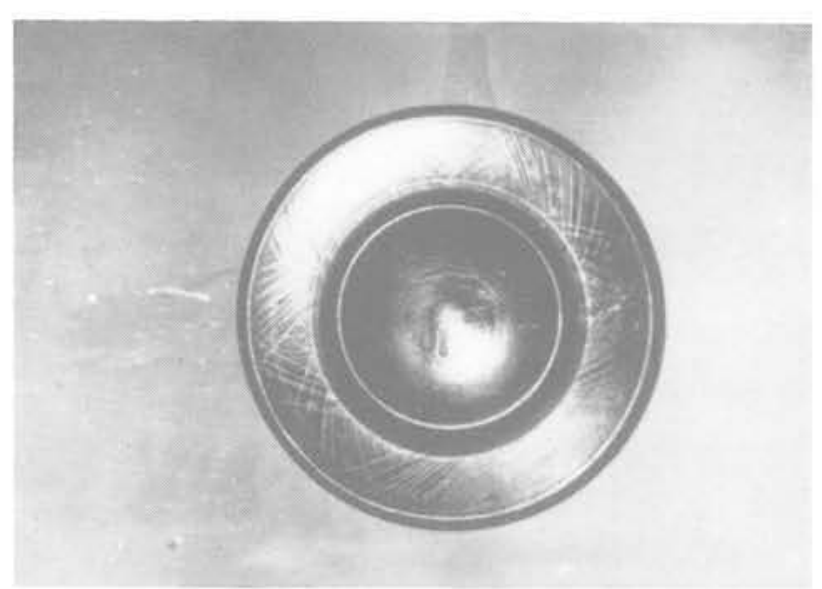

Fig. 11. - Multitude de lignes de Luders discrètes dans de l'acier doux embouti.

jusqu'à envahir complètement une pièce en champ homogène sous l'effet de l'écrouissage qui suit le palier plastique. Leur apparence sous forme d'une double multiplicité discrète sur la figure 11 montre que des lignes de glissement se seraient localisées en deux familles distinctes encore plus sûrement. On en verra d'autres exemples plus loin.

II faut insister sur le fait que l'allongement progressif d'une ligne de glissement unique lou de quelques lignes de glissement) est aussi en accord avec l'observation, même si cette conception est parfois occultée par certaines intuitions du calcul à la rupture. La méthode des tranches, le cercle de Fellenius, les spirales logarithmiques de Rendulic admettent en effet comme présupposé le mouvement entre les blocs rigides. En fait, la pratique du poinçonnement des fondations ou celle des très grands glissements naturels, monire que les "blocs" en mouvement sont susceptibles de grandes déformations. Pour des structures plus petites, comme des fondations en surface ou des murs de soutènement, en poussée comme en butée, on a même montré la progression de l'effort avec la progression de la longueur de la surface de glissement et le développement de la surface de rupture a été mis en évidence par de nombreux expérimentateurs et avec des techniques très différentes ( $\gamma$-densimétrie pour suivre le développement de la surface de glissement par sa dilatance, radiographie X d'un quadrillage de billes de plomb placées dans la masse, déformation d'un quadrillage en déformation plane derrière une vitre, récemment par Darve à Grenoble par l'observation photographique d'un quadrillage tracé à la surface d'un massif de rouleaux selon la méthode ScheebelliDantu, etc.). La figure 12, d'après Chazy et Habib (1961), montre le développement d'une famille de surfaces de glissement sous un large poinçon, dont la plus longue débouche juste en surface; il est nettement visible sur cette figure que le déplacement diminue lorsqu'on s'approche de la surface libre. Il est assez intuitif que les autres surfaces de glissement ont été arrêtées en cours de développement et qu'elles sont apparues après la surface principale qui les enveloppe toutes.

Les figures $13 \mathrm{a}$ et $13 \mathrm{~b}$ illustrent quelques cas où la formation de surfaces de glissement isolées peut être observée et d'autres où la déformation reste quasi homogène,

Le cas de l'expansion de la cavité circulaire au sein du massif (fig. 13b) ou près de la surface du massif (fig. 13a) montre nettement l'influence du ligament qui peut supporter ou non l'augmentation des contraintes dans le domaine élastique, c'est-à-dire ne permet pas ou au contraire qui permet le développement jusqu'à la surface libre d'une surface de glissement isolée.

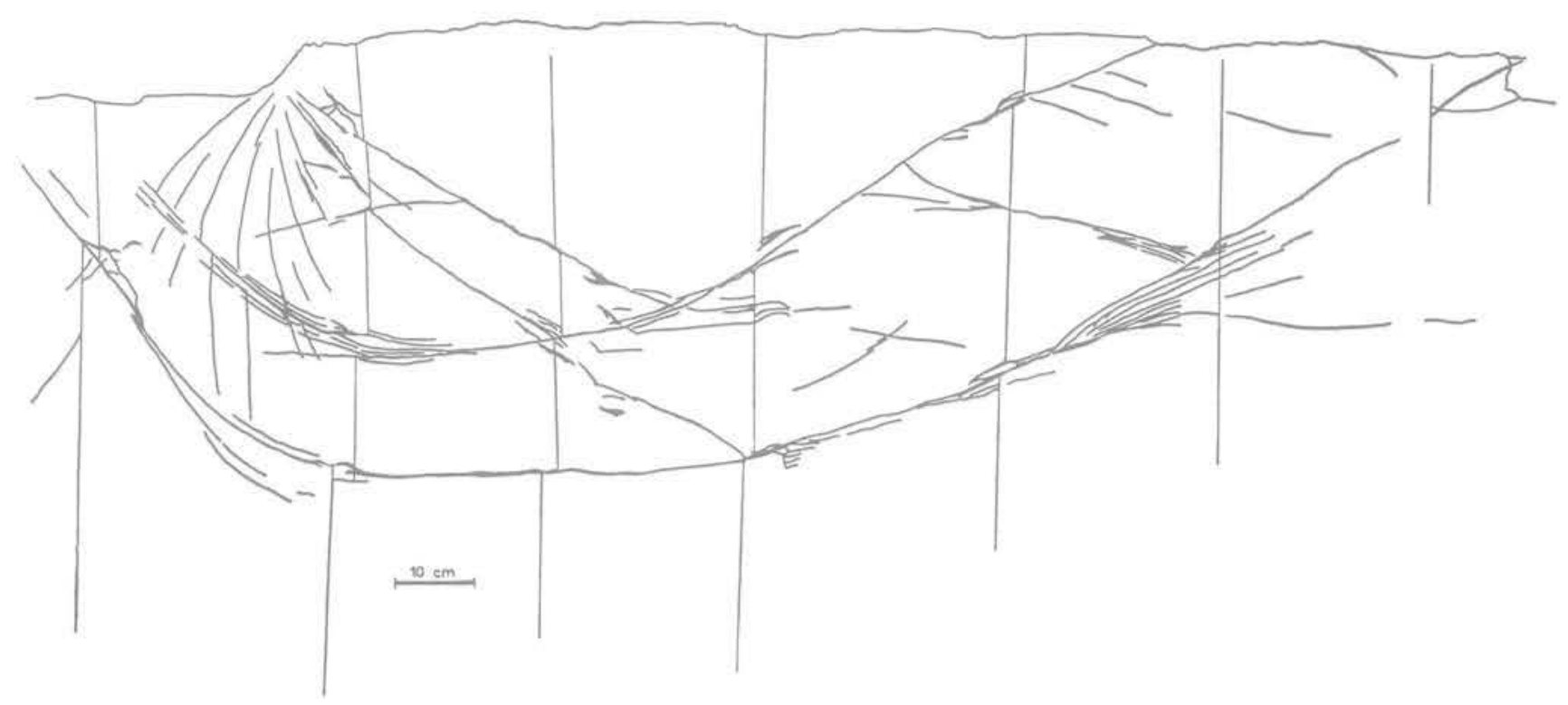

Fig. 12. - Surfaces de glissement sous une fondation. 


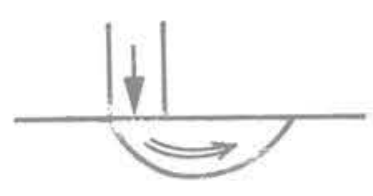

Poinçon superficiel

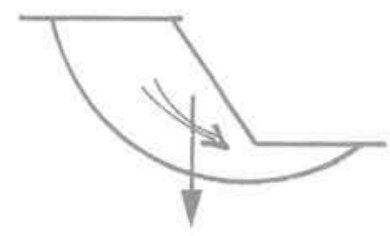

Glissement de terrain

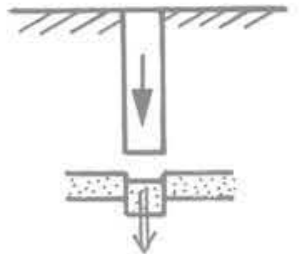

Poinçon profond

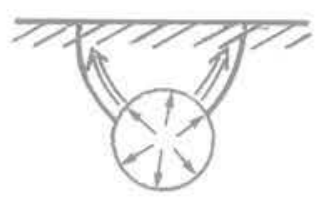

Expansion cylindrique près de la surface

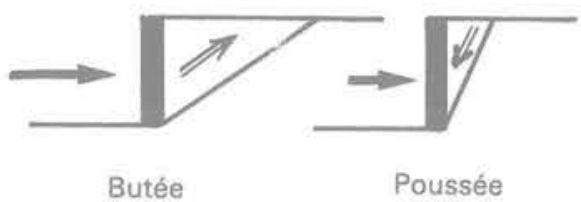

Butée

Poussée

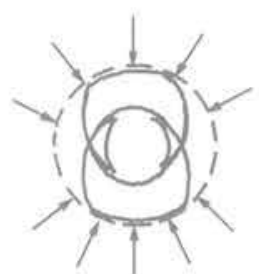

Contraction cylindrique

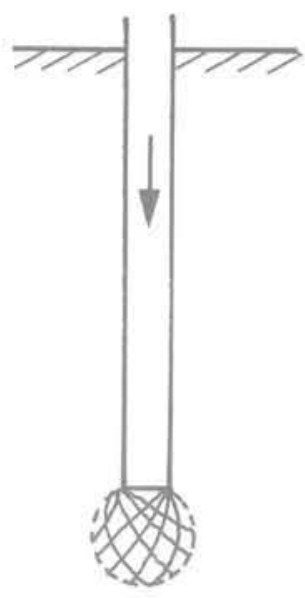

Pieu

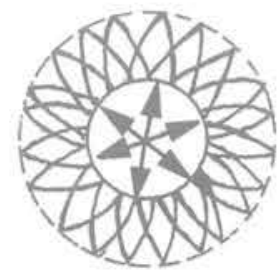

Expansion cylindrique loin de la surface

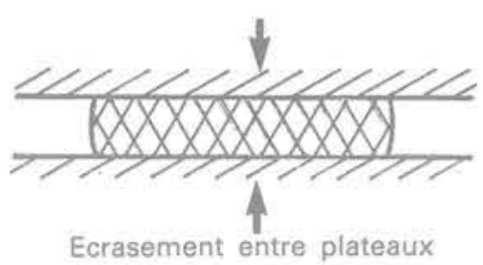

b. Déformation homogène

a. Surfaces de glissement

Fig. 13. - Différents cas de déformation.

(Les flèches noires indiquent les forces, les flèches claires, les déplacements).

\section{L'ESSAI DE CISAILLEMENT DIRECT}

Une façon classique de mesurer la résistance au cisaillement d'un sol est l'essai de cisaillement rectiligne à déplacement contrôlé et en particulier l'essai à la boîte de Casagrande. Ce mode d'essai semble actuellement un peu tombé en désuétude, sans qu'on en donne vraiment de bonnes raisons. En général, on reproche au cisaillement direct le fait qu'au cours de l'essai, les contraintes principales changent de direction, alors qu'elles restent fixes dans l'essai triaxial; de même, la contrainte principale intermédiaire dans le cisaillement direct est variable en cours d'essai, on n'en est pas maître et on ne la connaît pas alors que dans l'essai triaxial elle est égale à la plus petite des contraintes principales ou, éventuellement, à la plus grande dans un essai en extension. Les contraintes et, partant, les déformations ne sont peut-être pas toujours très homogènes dans les demi-boîtes qui accrochent 
l'échantillon. Il en résulte une quasi-impossibilité de mesurer les pressions interstitielles en cours de cisaillement, au contraire de l'essai triaxial. Enfin, bien que cette raison soit inavouable, un appareil triaxial est sans doute moins encombrant et moins onéreux qu'une machine de cisaillement... Les trois premiers motifs cités ne sont cependant pas très convaincants dans la mesure où la situation dans la boîte de cisaillement correspond souvent à ce qui se passe dans la réalité: au bord d'une grande fouille en cours de creusement, par exemple, la direction des contraintes principales varie avec l'avancement des travaux, on n'est pas maître de la contrainte principale intermédiaire et le champ de déformation n'est pas uniforme. L'essai de cisaillement (fig. 14) pose cependant un certain nombre

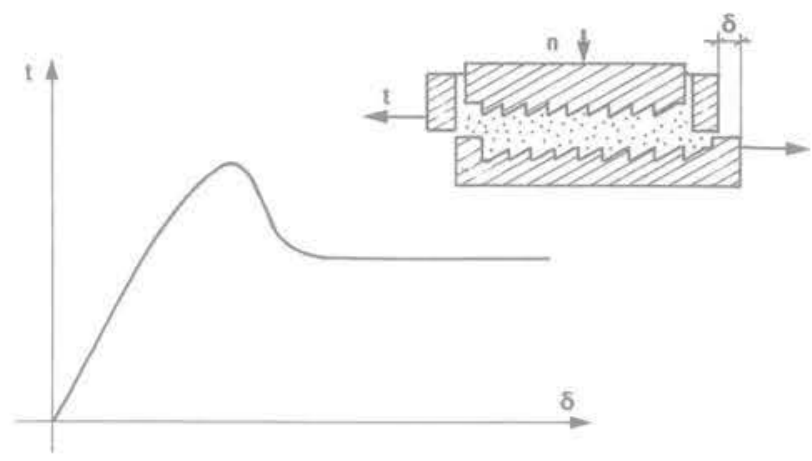

Fig. 14. - Essai de cisaillement direct.

de problèmes. Lorsque la courbe (effort de cisaillement-déplacement de la boîte) présente de l'écrouissage, on a du mal à identifier une surface de glissement dans la boîte et si on arrête l'essai en cours de route, on trouve une zone lenticulaire de déformation à la place du plan de rupture. $\mathrm{Si}$ la courbe présente un maximum et un palier permettant de définir $\varphi_{\mathrm{mx}}$ et $\varphi_{\mathrm{r}}$ pour un sable lou $\mathrm{t}_{\mathrm{mx}}$ et $\mathrm{t}_{\mathrm{r}}$ pour une argile), on constate que le maximum de résistance est atteint typiquement pour un déplacement de l'ordre de quelques millimètres dans la boîte de Casagrande classique et que la dilatance du plan de glissement est erratique, commençant tantôt à l'avant, tantôt à l'arrière ce qui se traduit par des mouvements de bascule différents avant d'obtenir la dilatance complète du plan de glissement. Enfin, la pente initiale de la courbe de cisaillement n'est guère compréhensible, et elle est d'autant plus ininterprétable en termes de module de glissement qu'on ne sait pas à quelle épaisseur de la "surface » de glissement la rapporter. Une expérience beaucoup plus pure est celle de la torsion sur cylindre. Nous rapportons sur la figure 15 les résultats de deux essais de torsion sur cylindre plein et sur cylindre plein entaillé horizontalement par une fissure sans épaisseur, effectués sur des éprouvettes en cire. Il s'agit d'un matériau écrouissable à $\varphi$ nul et par conséquent la localisation des déformations, si elle devait se produire, se ferait suivant un plan horizontal (et non sur un hélicoïde comme dans le cas $\varphi \neq 0$ ). La comparaison des deux courbes de rupture montre que tout s'est passé comme si on avait additionné la courbe élastique de torsion du cylindre plein (15a) et la courbe rigide plastique de la fissure sans épaisseur (15b). D'ailleurs,

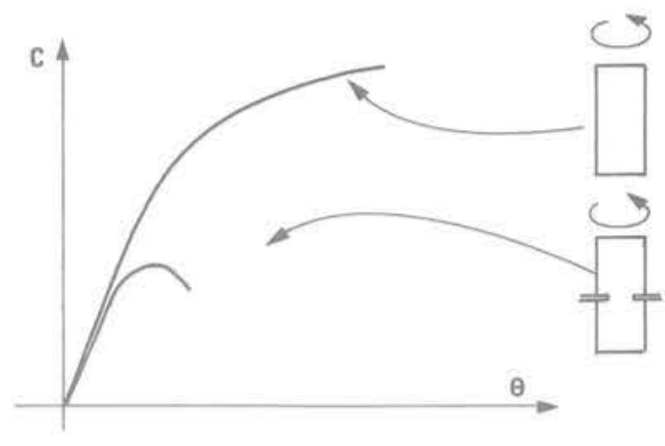

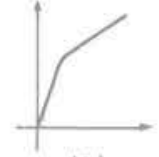

(a)
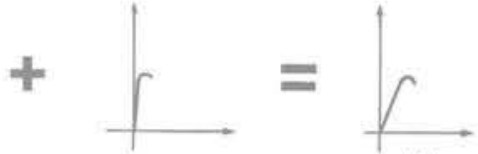

(b)
Fig. 15. - Essai de torsion sur éprouvette entaillée.

au cours des essais, les génératrices des éprouvettes cylindriques s'enroulaient en hélice autour de l'éprouvette; dans le cas de l'éprouvette entaillée, le rejet du glissement de part et d'autre de la fissure était pratiquement insensible avant la plastification de la section affaiblie: la rotation entre les lèvres de la fissure est restée inexistante pendant l'essai de torsion, ce qui est normal puisque l'épaisseur du plan de glissement était infiniment petite; des mouvements relatifs ne se sont produits qu'à l'approche immédiate de la rupture, et la forme de la courbe après le seuil correspond vraisemblablement à l'écrouissage cinématique engendré par la progression de la plastification de la périphérie de l'éprouvette vers le centre, dans une déformation que la cinématique rend non uniforme.

La comparaison entre cet essai de torsion et l'essai de cisaillement n'est pas immédiate puisque la progression de la fissure en torsion correspond à une propagation en mode III alors qu'au cisaillement direct correspond une progression en mode II, mais l'analogie est évidente, et l'interprétation de la pente initiale de la courbe effort-déformation de l'essai de cisaillement à la boîte de Casagrande devient très simple: il se produit une progression en mode II de deux fissures issues des bords antérieur et postérieur de l'échantillon et qui se dirigent vers le milieu de l'échantillon. Les basculements erratiques de la demi-boîte supérieure correspondent à des vitesses de progression inégales fonction de la qualité du remplissage initial de la boîte. La pente du début de la courbe effort-déplacement correspond à la progression irréversible de cette plastification et non à un quelconque phénomène d'élasticité. Reprenant le schéma de la figure 10 et en l'appliquant à l'essai de Casagrande, on peut donner un diagramme de distribution de la contrainte de cisaillement dans le plan horizontal (fig. 16). Ce schéma est sans doute d'autant plus représentatif de la réalité que l'échantillon est épais. Les dents des pierres poreuses supérieures et inférieures ont évidemment pour but de transmettre le plus uniformément possible la déformation dans le plan de glissement. Il est probable cependant que l'effet des bords d'attaque des boîtes est important. 


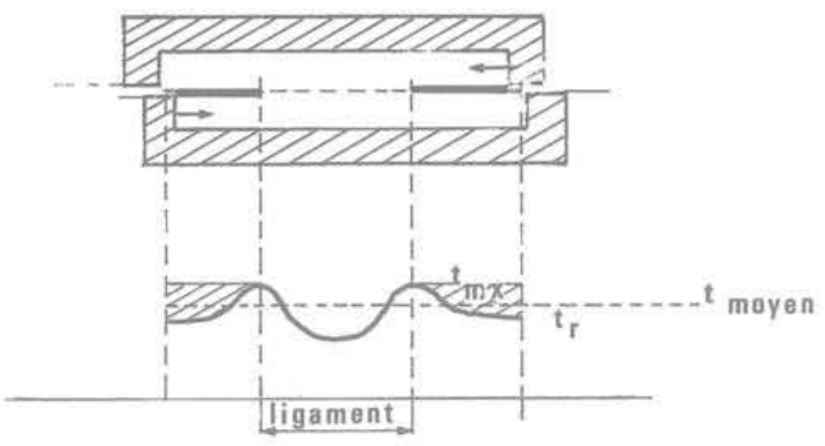

Fig. 16. - Distribution de la contrainte de cisaillement dans le plan de rupture d'un essai de cisaillement direct.

On voit en particulier que la valeur moyenne de la contrainte, qui est en définitive la seule grandeur mesurée, ne correspond pas à la résistance maximale du sol et qu'il existe certainement un effet d'échelle dans cet essai: la progression de la rupture dans une boîte très grande donnerait un effort moyen se rapprochant de plus en plus de la valeur résiduelle; on peut imaginer une série d'échantillons de plus en plus minces donnant des courbes effort-déplacement emboîtées, l'échantillon d'épaisseur nulle donnant une courbe effort-déplacement se rapprochant du schéma rigide-plastique et étant évidemment le plus représentatif (fig. 17).

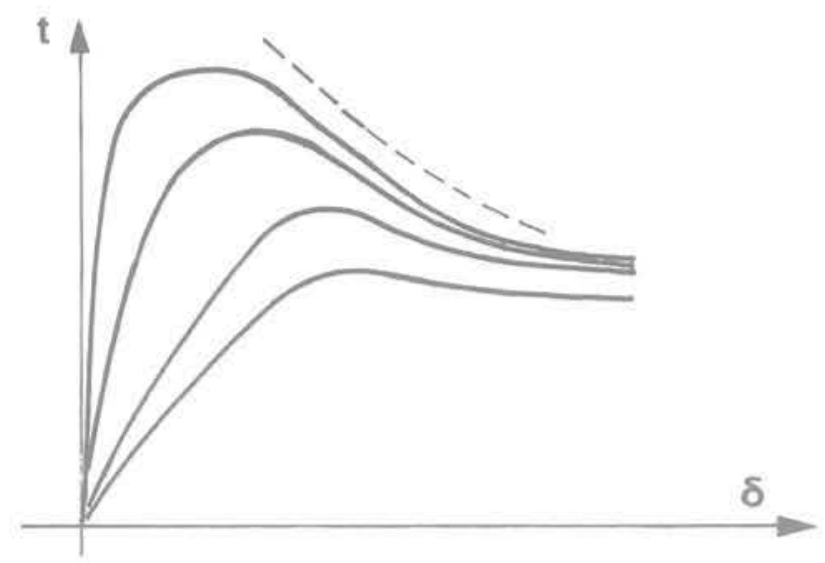

Fig. 17. - Recherche d'une courbe de cisaillement directe idéale.

On voit par contre que le cisaillement linéaire après un certain déplacement donne parfaitement la valeur de la résistance résiduelle $\mathrm{t}_{\mathrm{r}}$ : lorsque les deux fissures se sont rejointes et que les deux bosses du diagramme de cisaillement de la figure 16 se sont effacées, la valeur de $t_{\text {moven }}$ est bien confondue avec $t_{r}$. L'essai de cisaillement linéaire est parfaitement adapté à la mesure de la résistance résiduelle.

\section{L'ESSAI TRIAXIAL}

On peut se demander si la détermination de la résistance maximale $t_{m x}$ par un essai triaxial ne participe pas de la même critique que celle de l'essai de cisaillement linéaire. Dans la mesure où le champ de contraintes est uniforme, la contrainte de cisaillement sur un plan de glissement potentiel est constante. Admettant l'existence d'un point faible dans ce plan, le schéma de la figure 10, adapté à ce cas sur la figure 18, montre que le déficit de résistance ne peut être supporté par le ligament et que la rupture devrait être de type fragile dès l'apparition de la première amorce, ce qui n'est pas.
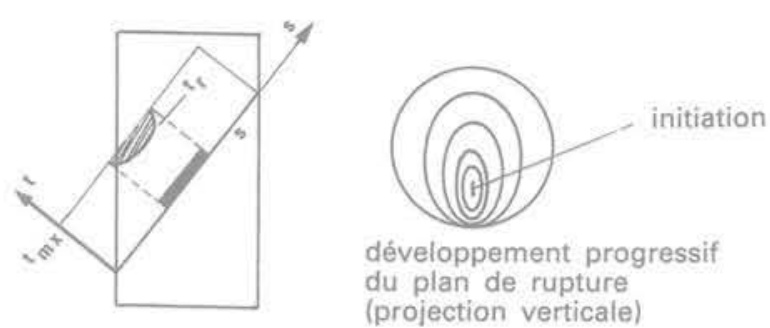

développement progressif du plan de rupture (projection verticale)

Fig. 18. - Progression de la rupture dans une éprouvette d'essai triaxial.

II est bien plus logique et plus conforme à l'expérience d'admettre le développement d'un plan de glissement à partir d'un point faible situé près de la singularité de la base; ce développement se produit probablement par auréoles successives, comme pour n'importe quelle entaille, et cela explique la formation d'un ou d'un nombre limité de plans de glissement et non d'un cône de rupture comme on en observe sur les matériaux fragiles avec endommagement, comme les roches ou les bétons en compression simple. La valeur maximale de cette résistance qui se développe progressivement à la compression est donc dans l'essai triaxial sujette à la même critique que celle de l'essai de cisaillement. Certes, les essais sont parfaitement répétitifs, mais la valeur maximale ainsi mesurée correspond-elle vraiment à la résistance de la matière?

Pour s'en assurer, nous avons réalisé des essais triaxiaux sur échantillons courts. Dans un essai triaxial courant on utilise des éprouvettes d'élancement compris entre 2 et 2,5 de façon que la partie centrale soit éloignée du frettage des bases et pour laisser la possibilité du développement libre d'un plan de glissement (fig. 1). Par exemple, on peut tolérer un élancement plus petit de l'ordre de 1,5 pour des éprouvettes d'argile du sparnacien de Paris où le plan de rupture est incliné à près de $45^{\circ}$ que pour des éprouvettes d'argile du sannoisien où le plan de rupture est incliné à environ $30^{\circ}$ sur l'axe de l'éprouvette. Mais, si on pouvait imposer des appuis sans frottement, rien ne s'opposerait à l'emploi d'éprouvettes plus courtes. Nous allons montrer que cette affirmation doit être limitée aux matériaux écrouissables ou tout au moins à la partie située avant le maximum de la courbe effort-déformation des matériaux radoucissants, 
De nombreux expérimentateurs ont cherché à réaliser des appuis sans frottement pour les sols, pour les bétons ou pour les roches. La solution que nous avons retenue pour le sable a consisté à interposer entre l'échantillon et l'acier des têtes d'appui une mince feuille de caoutchouc reposant sur un film de graisse (fig. 19a); cette solution n'est pas parfaite, mais représente une bonne approximation d'une contrainte normale à la surface de contact.
Les résultats obtenus ont été les suivants. Au cours de l'écrasement d'une éprouvette courte de sable, le matêriau est expulsé latéralement et il se forme un bourrelet autour du cylindre initial qui perturbe probablement la fin de l'essai (fig. 19b), c'est-à-dire lorsque la déformation dépasse 15 ou $20 \%$. Lorsque le sable est dense, on voit apparaitre sur le bourrelet une multitude de lignes de glissement.
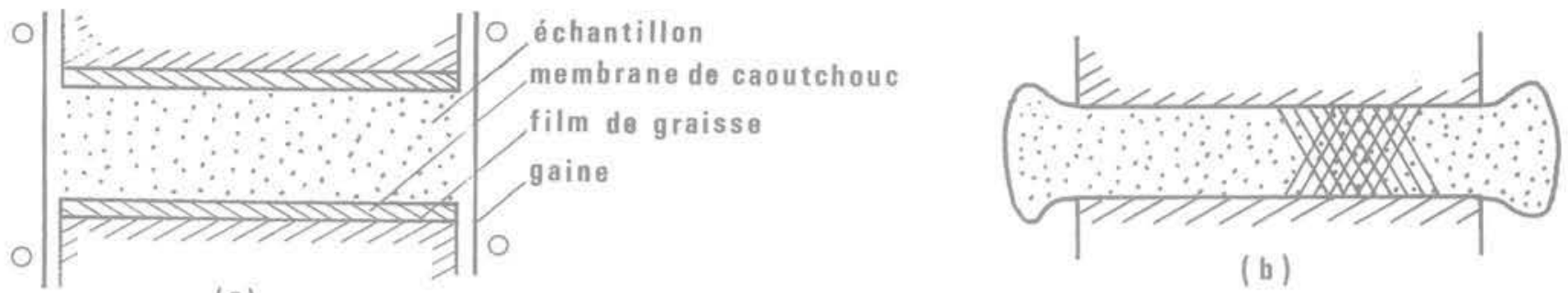

(a)

Fig. 19. - Essai triaxial sur échantillon court.

Les courbes effort-déformation des échantillons courts de sable dense ne montrent pas de radoucissement pour des déformations élevées alors que celles des échantillons longs de même densité présentent un maximum suivi d'un radoucissement, accompagné de l'apparition d'un plan de glissement isolé, c'est-à-dire avec localisation de la déformation. Mais ce qui était particulièrement intéressant dans ces essais était le résultat quantitatif suivant: la valeur du maximum de résistance des échantillons longs était égale à la valeur du palier de l'échantillon court (fig. 20).

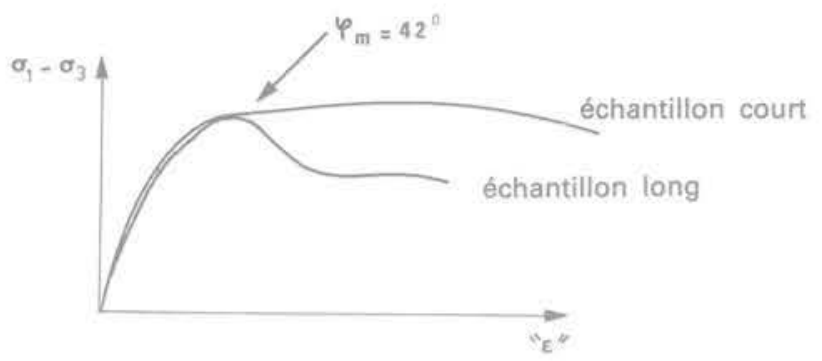

Fig. 20. - Essais triaxiaux sur échantillon court et sur échantillon long de sable.

Des essais d'écrasement d'échantillons courts ont été aussi effectués sur une argile très peu plastique provenant d'un prélèvement à grande profondeur. Bien que ce matériau présentait en compression simple une localisation de la déformation sur un plan de rupture (fig. 21), son comportement était pratiquement fragile, c'est-à-dire avec une perte quasi-complète de résistance après la rupture. Compte tenu de la raideur du matériau, on a cherché à éviter la présence d'un film de graisse au contact de l'argile, susceptible d'être expulsée par la pression et d'engendrer des tractions

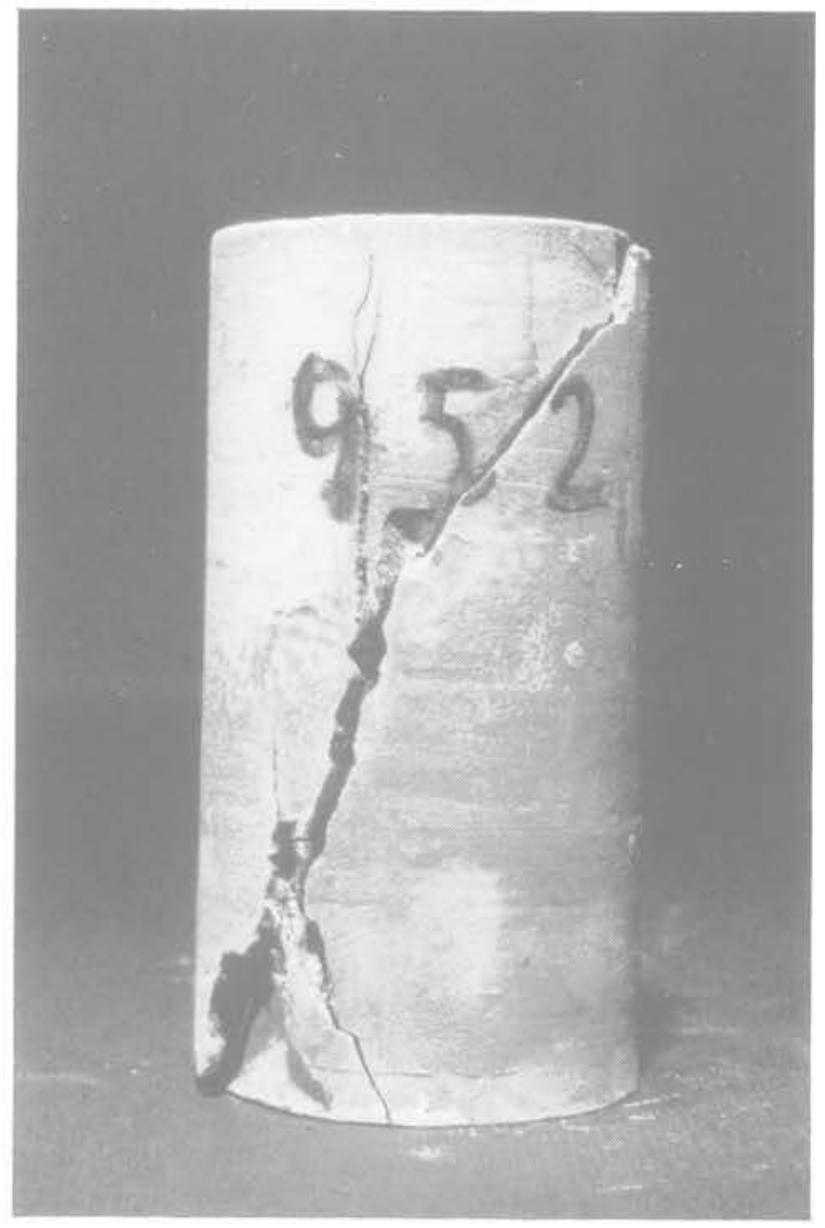

Fig. 21. - Localisation de la déformation dans une éprouvette longue d'argile raide. 
sur les plans passant par l'axe de l'éprouvette et on a utilisé un autre mode d'antifrettage des têtes des échantillons courts. Il était constitué par des couches de confettis d'aluminium lubrifiés par de la graisse ou par du bisulfure de molybdène. Le contact (argileconfettis d'aluminium) n'apporte pas une résistance à la traction, donc pas de frettage de la base de l'éprouvette; le lubrifiant permet d'avoir une contrainte normale aux confettis. La rupture des échantillons courts s'est produite avec apparition d'une multitude de cassures et est présentée sur la figure 22 où l'on voit nettement le dispositif d'antifrettage. Le résultat des essais est indiqué sur la figure 23: il existe encore un certain radoucissement après la rupture de l'échantillon court, mais la valeur des maximums est pratiquement la même entre les échantillons long et court avec antifrettage.

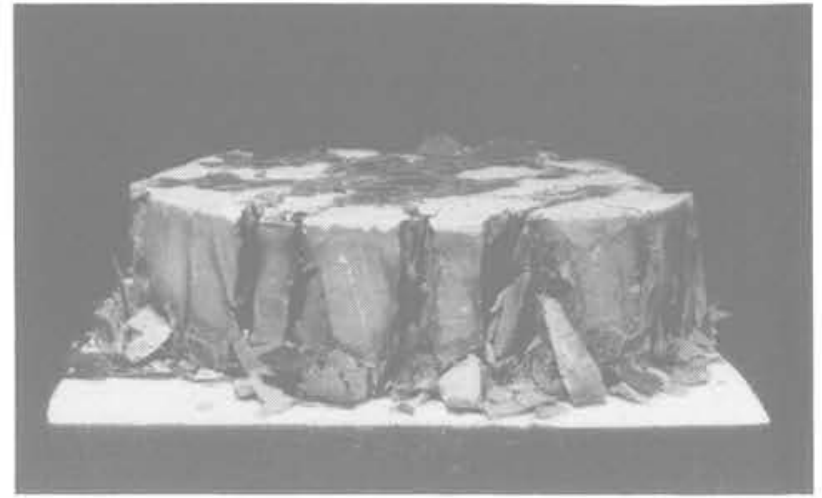

Fig. 22. - Multitude de cassures dans une éprouvette courte d'argile raide.

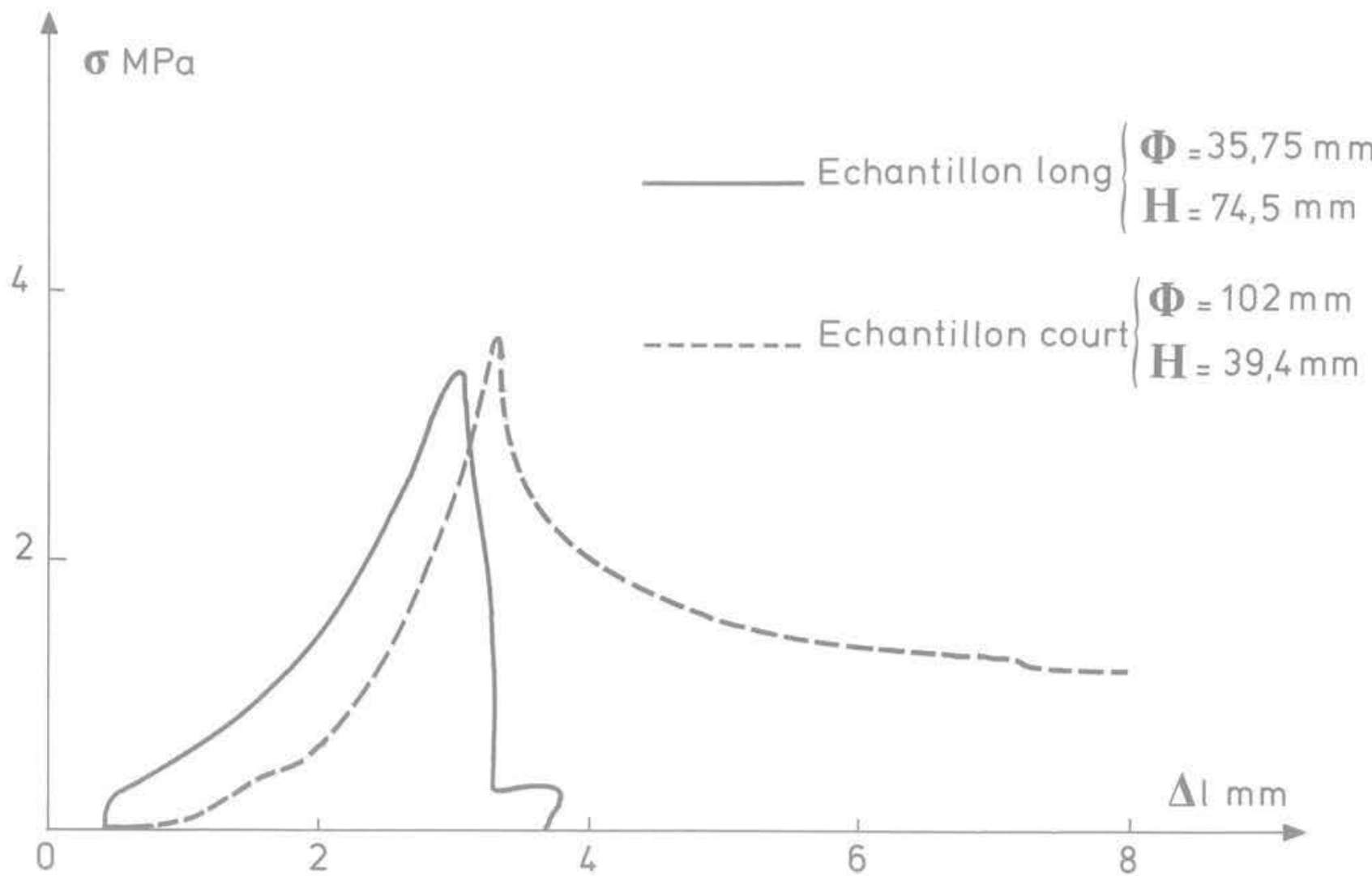

Fig. 23. - Essais de compression simple sur échantillon court et sur échantillon long d'argile.

Ce résultat est évidemment différent de ce que l'on aurait avec des échantillons courts et frottement aux appuis. On sait dans ce cas que la résistance est beaucoup plus grande (Hudson, Brown, Fairhurst, 1971).

Pour les sables et pour les argiles, il apparaît ainsi que la valeur de la résistance maximale obtenue à l'appareil triaxial est indépendante de la forme de l'échantillon: elle correspond donc à une propriété intrinsèque de la matière. Par contre, la signification physique de la pente de la courbe de radoucissement demeure incertaine.

Après avoir jeté, puis levé un doute sur la valeur de la résistance maximale mesurée dans un essai triaxial et par conséquent sur la valeur de $\varphi_{\mathrm{mx}}$ d'un sable, montrons maintenant que la valeur de $\varphi_{\mathrm{r}}$ dans l'essai triaxial est inexacte et mérite généralement d'être corrigée. On sait que dans un essai triaxial en compression sur du sable, l'angle $\beta$ de la surface de glissement avec l'axe de l'éprouvette est d'autant plus 


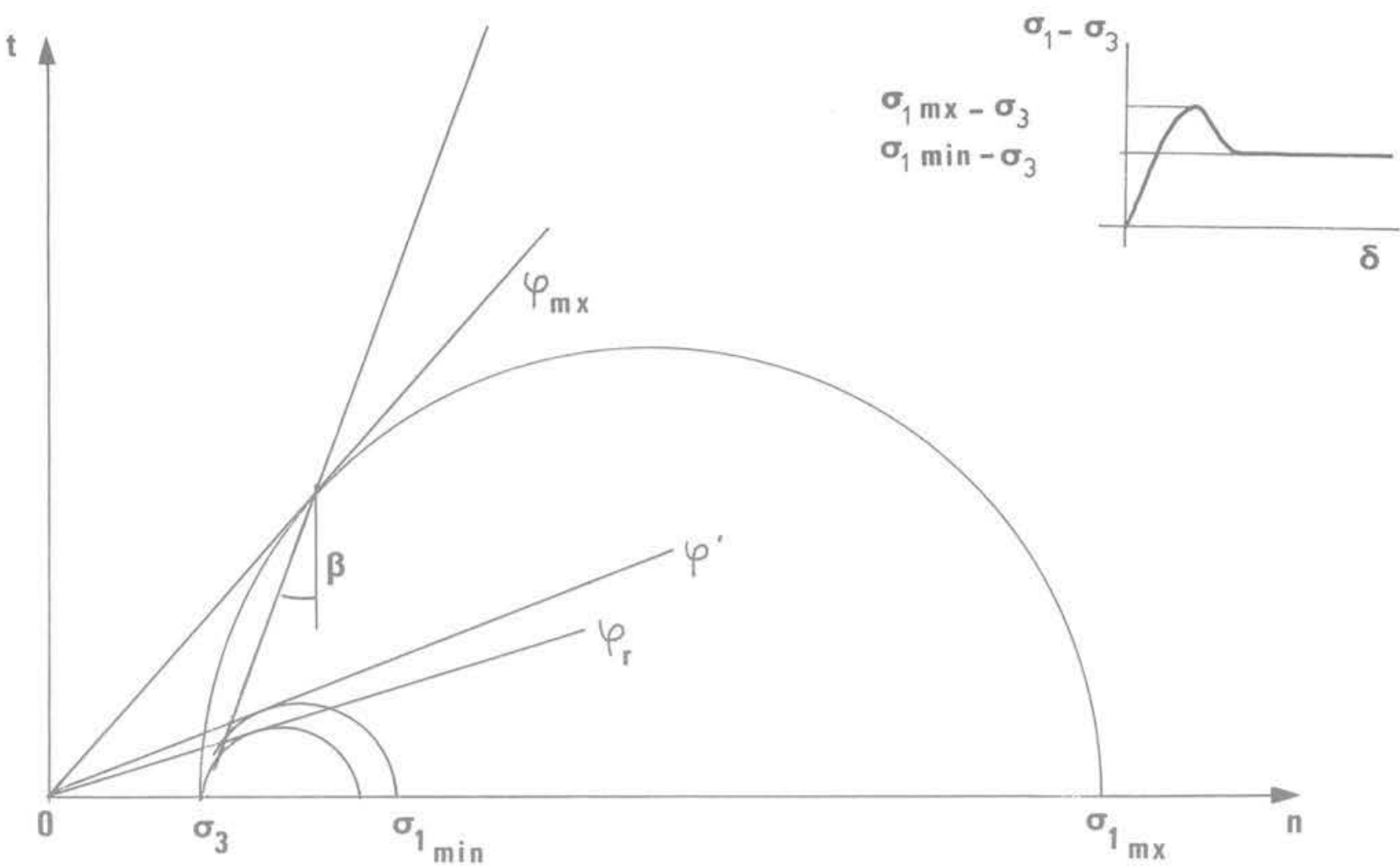

Fig. 24. - Cercles de Mohr du frottement maximal et du frottement résiduel.

petit que l'angle de frottement interne $\varphi$ est grand; on peut dire que $\beta$ est d'autant plus petit que la densité du sable est grande ou que la dilatance est forte. Sans vouloir prendre parti sur l'origine physique des lignes de glissement et simplement pour pouvoir mener un calcul à son terme, admettons, après Coulomb, la relation $\beta=\frac{\pi}{4}-\frac{\varphi}{2}$ (ce qui signifie que la ligne de glissement est une ligne caractéristique des contraintes). Après le radoucissement, lorsque la dilatance est achevée, l'angle de frottement résiduel $\varphi_{\mathrm{r}}$ dans le plan de glissement correspond par exemple à la valeur critique $\varphi_{c}$ mais la direction du glissement est toujours $\beta$. Le cercle de Mohr correspondant au glissement sur ce plan est donc plus grand que ce qu'il serait si l'éprouvette était homogène d'angle de frottement $\varphi_{\mathrm{r}}$. $\mathrm{La}$ figure 24 indique les différentes grandeurs. $\mathrm{La}$ résistance maximale sur la courbe contraintedéplacement définit $\sigma_{1 \mathrm{mx}}-\sigma_{3}$ d'où $\varphi_{\mathrm{mx}}$, la résistance correspondant au palier de l'essai définit $\sigma_{1 \min }-\sigma_{3}$ d'où un angle $\varphi^{\prime}$ qui est plus grand que la résistance résiduelle $\varphi_{\mathrm{r}}$.

Un calcul simple bien qu'un peu long permet d'obtenir la relation:

$\operatorname{tg}^{2}\left(\frac{\pi}{4}+\frac{\varphi^{\prime}}{2}\right)=\frac{\operatorname{tg} \varphi_{\mathrm{r}}\left[\operatorname{tg}^{2}\left(\frac{\pi}{4}+\frac{\varphi}{2}\right)+1\right]}{\operatorname{tg}\left(\frac{\pi}{4}+\frac{\varphi}{2}\right)-\operatorname{tg} \varphi_{\mathrm{r}}}$
L'abaque de la figure 25 permet de calculer la correction $\varphi^{\prime}-\varphi_{r}$ qu'il faut apporter à la valeur $\varphi^{\prime}$ mesurée pour déterminer la valeur de $\varphi_{\mathrm{r}}$.

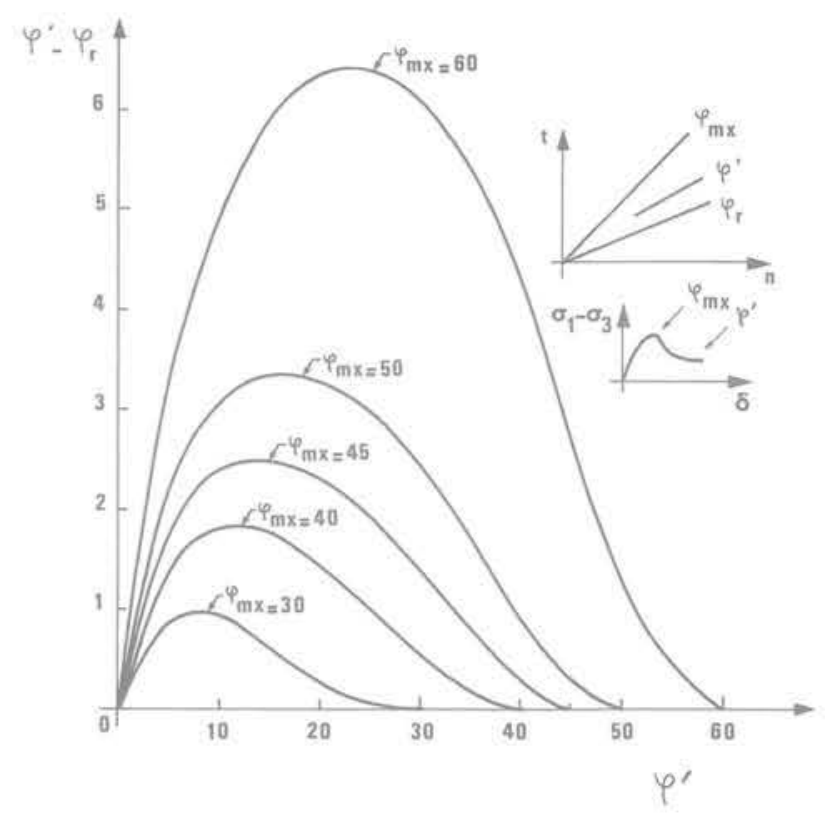

Fig. 25. - Correction $\left(\varphi^{\prime}-\varphi_{r}\right)$ pour le frottement résiduel (essai triaxial). 
Cette correction est de quelques degrés dans les cas courants de la mécanique des sols; elle peut devenir très importante en mécanique des roches pour les grands angles de frottement maximal.

\section{APPLICATIONS PRATIQUES}

On vient de voir qu'au laboratoire de mécanique des sols, la correction à apporter à l'essai triaxial pour déterminer $\varphi_{\mathrm{r}}$ est petite, de l'ordre de 1 ou $2^{\circ}$, lorsqu'un plan de glissement s'est fixé dans l'éprouvette.

Le même phénomène peut avoir une importance beaucoup plus grande lorsque les surfaces de glissement ne sont pas planes et que les champs de contraintes ne sont pas uniformes. Prenons l'exemple de la force portante des fondations superficielles sur des matériaux pulvérulents. Le glissement généralisé se produit sur une surface de rupture dont l'orientation et le tracé sont fonction de $\varphi_{\mathrm{mx}}$ (ou de la dilatance du sable correspondant à $\varphi_{\mathrm{mx}}$ peu importe). Mais progressivement à la localisation le frottement se rapproche de $\varphi$ critique. De sorte qu'on peut simplement dire que la force portante limite $\mathrm{q}_{\mathrm{u}}$, calculée par la formule classique du terme de surface $\gamma \frac{B}{2} N_{\gamma}$, est encadrée par les deux valeurs:

$$
\gamma \frac{\mathrm{B}}{2} \mathrm{~N}_{\gamma}\left(\varphi_{\mathrm{c}}\right)<q_{\mathrm{u}}<\gamma \frac{\mathrm{B}}{2} \mathrm{~N}_{\gamma}\left(\varphi_{\mathrm{mx}}\right)
$$

Pour prendre un exemple numérique, les tables de Caquot-Kerisel (1956) donnent $N_{\gamma}\left(\varphi_{c}=32^{\circ}\right)=31$ et $N_{\gamma}\left(\varphi_{m x}=40^{\circ}\right)=114$, ce qui montre l'ordre de grandeur de l'incertitude théorique lié au phénomène du radoucissement.

Il est évidemment extrêmement difficile de resserrer cet encadrement par une approche théorique, étant donné le caractère discontinu de la déformation. On peut toutefois proposer des approximations.

Examinons d'abord la borne supérieure. Il est bien clair que dans la surface de glissement (ou dans les surfaces de glissement) l'angle de frottement interne $\varphi_{c}$ est rapidement atteint et que $\varphi_{\mathrm{mx}}$ n'est sans doute représentatif que de l'état des blocs rigides. Par analogie avec la façon de calculer utilisée dans la méthode des cercles de glissement, c'est-à-dire en prenant la résistance au cisaillement de la surface de rupture, on peut envisager de minorer la valeur de la borne supérieure par le coefficient $\frac{\operatorname{tg} \varphi_{c}}{\operatorname{tg} \varphi_{\max }}$ pour ramener le frottement à sa véritable valeur.

Pour la borne inférieure, on remarquera de la même façon que si l'angle de frottement $\varphi_{\mathrm{c}}$ correspond bien à la résistance résiduelle, par contre la ligne de glissement est trop courte. On peut donc envisager un coefficient majorateur qui soit fonction de l'extension de la surface de glissement. Rappelons que la largeur $\mathrm{L}$ du refoulement latéral est proportionnelle à la largeur B de la fondation. D'après le réseau classique des lignes caractéristiques des contraintes, on peut écrire:

$$
\frac{L}{B}=\operatorname{tg}\left(\frac{\pi}{4}+\frac{\varphi}{2}\right) e^{\frac{\pi}{2} \operatorname{tg} \varphi}
$$

Il reste à définir quelle fonction de $\mathrm{L}$ choisir pour le coefficient majorateur. Toutefois par analogie avec le calcul des cercles de glissement, on peut penser que $\mathrm{L}$ intervient deux fois, la première pour définir la longueur de l'arc résistant, la deuxième pour définir la contrainte normale à la surface de glissement. Bien entendu, deux surfaces de glissement ne sont pas homothétiques mais la bonne fonction de correction est sans doute plus proche de $\mathrm{L}^{2}$ que de $\mathrm{L}$. De sorte qu'on peut proposer un coefficient majorateur de la borne inférieure de la forme:

$$
\left(\frac{L\left(\varphi_{m x}\right)}{L\left(\varphi_{c}\right)}\right)^{2}=\frac{\operatorname{tg}^{2}\left(\frac{\pi}{4}+\frac{\varphi_{m x}}{2}\right) e^{\pi t g \varphi m x}}{\operatorname{tg}^{2}\left(\frac{\pi}{4}+\frac{\varphi_{c}}{2}\right) e^{\pi t g \varphi c}}
$$

Les deux corrections proposées sont indiquées sur la figure 26: elles fournissent des résultats voisins, ce qui n'a pas pouvoir de démonstration mais donne tout de même une certaine confiance dans l'approximation obtenue. Rappelons que Meyerhof (1961) avait donné pour le terme de surface une formule dite semiempirique, c'est-à-dire sans aucune prétention théorique mais s'accordant avec des calculs par la méthode des cercles de glissement, dont l'expression était:

$$
\begin{aligned}
& N_{\gamma}=\left(N_{a}-1\right) \operatorname{tg} 1,4 \varphi \\
& =\left[\operatorname{tg}^{2}\left(\frac{\pi}{4}+\frac{\varphi}{2}\right) e^{\pi t g \varphi}-1\right] \operatorname{tg} 1,4 \varphi
\end{aligned}
$$

il n'est donc pas étonnant que les deux corrections proposées donnent un accord satisfaisant.

On peut envisager la même approche pour d'autres problèmes de mécanique des sols et proposer des corrections aux formules classiques pour les matériaus avec radoucissement.

Prenons, par exemple, le cas de la poussée et de la butée normale à un écran vertical soutenant un massif horizontal de matériau pulvérulent. Les coefficients de butée et de poussée sont:

$$
K_{\mathrm{p}}=\operatorname{tg}^{2}\left(\frac{\pi}{4}+\frac{\varphi}{2}\right) \text { et } K_{\mathrm{a}}=\operatorname{tg}^{2}\left(\frac{\pi}{4}-\frac{\varphi}{2}\right)
$$

En butée, lorsque le sable est suffisamment dense pour qu'une surface de glissement apparaisse on peut écrire l'encadrement:

$$
\operatorname{tg}^{2}\left(\frac{\pi}{4}+\frac{\varphi_{c}}{2}\right)<K_{p}<\operatorname{tg}^{2}\left(\frac{\pi}{4}+\frac{\varphi_{m x}}{2}\right)
$$

On peut proposer le même coefficient minorateur de la borne supérieure que précédemment, soit:

$$
\operatorname{tg}^{2}\left(\frac{\pi}{4}+\frac{\varphi_{\mathrm{mx}}}{2}\right) \times \frac{\operatorname{tg} \varphi_{c}}{\operatorname{tg} \varphi_{\mathrm{mx}}}
$$

et pour la borne inférieure un coefficient fonction de la longueur $\mathscr{L}=H / \cos \left(\frac{\pi}{4}+\frac{\varphi}{2}\right)$ (H étant la hau- 


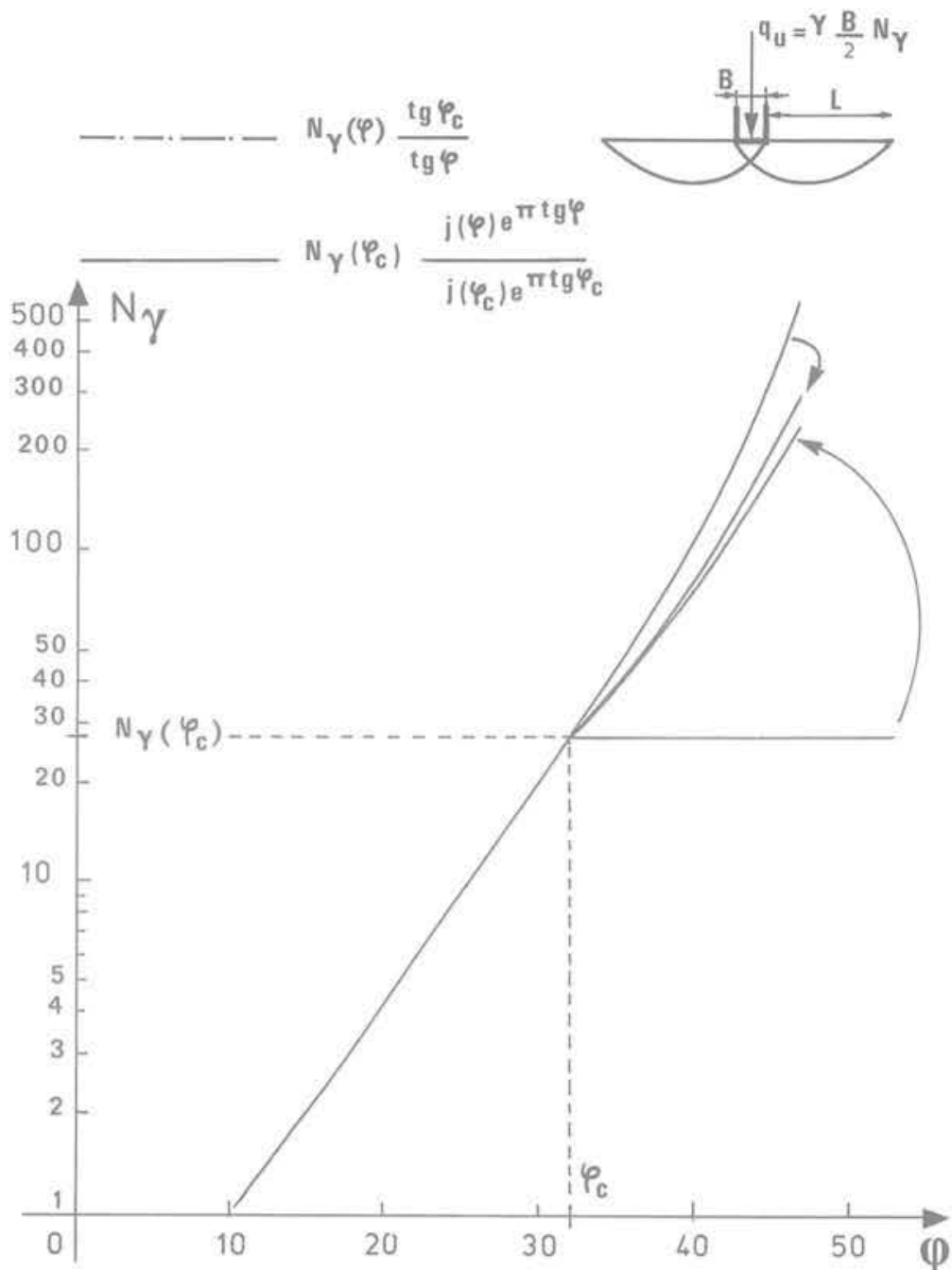

Fig. 26. - Corrections proposées pour la force portante des fondations superficielles sur un matériel pulvérulent d'angle de frottement interne $\varphi$.

teur du mur de soutènement). Mais, on prendra ici simplement une fonction linéaire de $\mathscr{L}$ car la contrainte normale à la surface de glissement n'a aucune raison d'être proportionnelle à $\mathscr{L}$ puisque la profondeur du plan de glissement est fixée par le pied du mur; on peut donc envisager un coefficient majorateur de la forme:

$$
\frac{\mathscr{L}\left(\varphi_{\mathrm{mx}}\right)}{\mathscr{L}\left(\varphi_{\mathrm{c}}\right)}
$$

et comme correction:

$$
\operatorname{tg}^{2}\left(\frac{\pi}{4}+\frac{\varphi_{c}}{2}\right) \times \frac{\cos \left(\frac{\pi}{4}+\frac{\varphi_{c}}{2}\right)}{\cos \left(\frac{\pi}{4}+\frac{\varphi_{\text {mx }}}{2}\right)}
$$

Les deux corrections proposées sont indiquées sur la figure 27. Elles sont encore assez proches l'une de l'autre pour fournir une approximation pratique suffisante.
La transposition au cas de la poussée est évidemment immédiate en changeant $\left(\frac{\pi}{4}+\frac{\varphi}{2}\right)$ en $\left(\frac{\pi}{4}-\frac{\varphi}{2}\right)$

\section{CONCLUSIONS}

Les considérations qui ont été exposées ci-dessus montrent que les phénomènes de localisation des déformations dans une ou plusieurs surfaces de glissement lors de la rupture des sols, ont des conséquences importantes, tant en laboratoire que pour la pratique des travaux. Une correction a été proposée pour la détermination de la résistance au cisaillement résiduelle dans l'essai triaxial, mais cet essai est surtout intéressant pour la détermination de la résistance maximale. De même, l'essai de cisaillement direct, qu'il soit rectiligne ou de torsion, est surtout utile et peut-être même irremplaçable pour la détermination de la résistance résiduelle. Dans la pratique, l'apparition de 


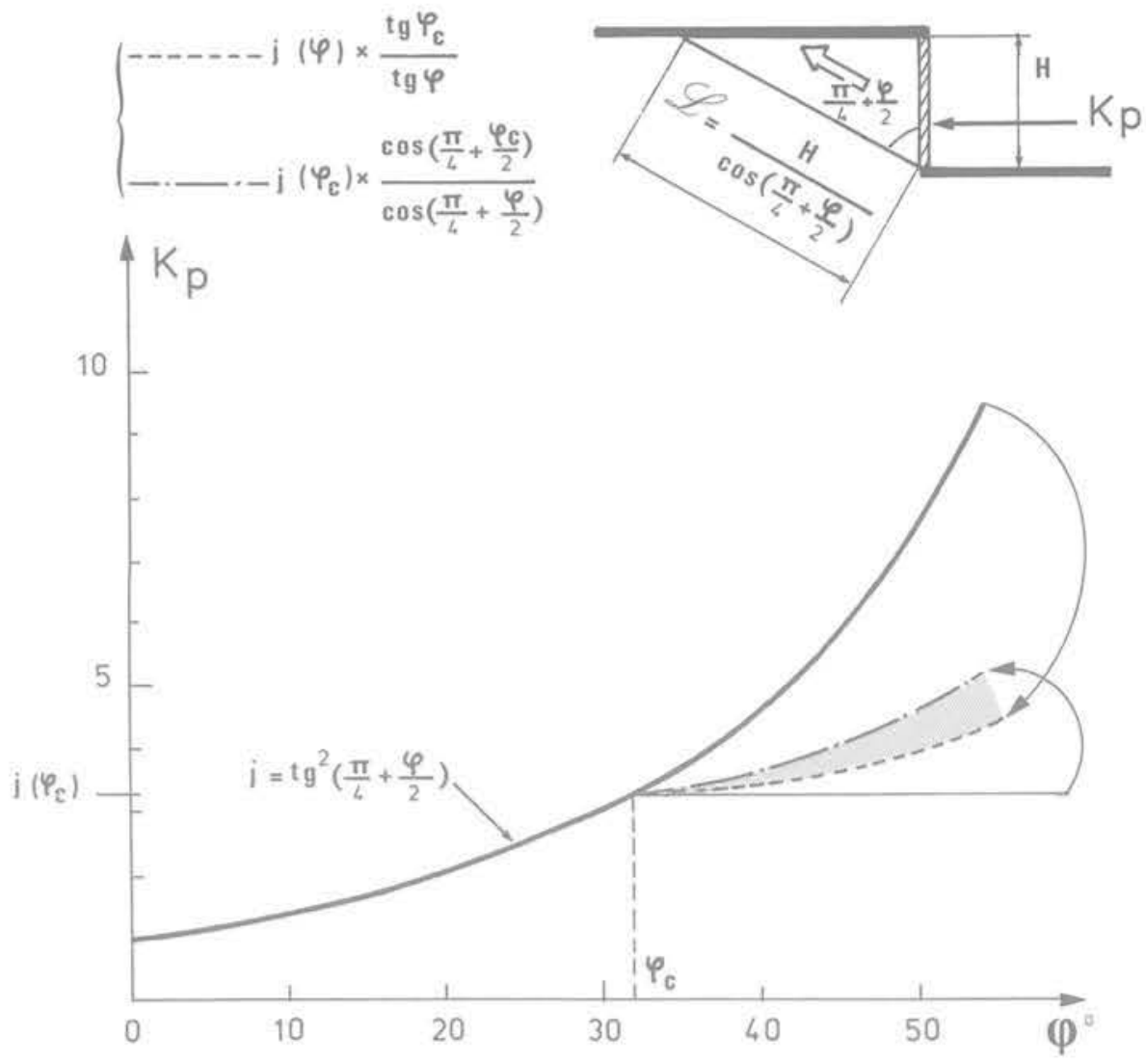

Fig. 27. - Corrections proposées pour la butée normale $K_{p}$ sur un mur soutenant un massif de sable d'angle de frottement interne $\varphi$.

discontinuités lors de la rupture d'un massif de terre provoque des perturbations importantes par rapport aux schémas classiques et modifie les valeurs calculées à partir de l'hypothèse de l'homogénéité de la déformation. Les corrections proposées ici pour le poinçon en surface et pour la butée sont évidemment de grossières approximations qui mériteraient d'être précisées dans les cas examinés, mais aussi qui méritent d'être généralisées à d'autres applications courantes de la pratique des fondations.

\section{BIBLIOGRAPHIE}

CAQUOT, A.; KERISEL, J. (1956). - Traité de mécanique des sols. - Gauthier-Villars, Paris, ch. XVI, p. 389.

CHAZY, C, HABIB, P. (1961). - Les piles du quai de Floride. $-5^{e}$ Congrès Int. de Méc. des Sols, Paris, juillet 1961, Com. 6/27, p. 669.

DARVE, F.; DESRUES, J.; JACQUET, M. (1980). Les surfaces de rupture en Mécanique des sols en tant qu'irréversibilité de déformation. - Cahiers du G.F.R., V.3, janvier 1980.
GOGUEL, J. (1983). - Etude mécanique des déformations géologiques. - B.R.G.M. Orléans, Manuels et Méthodes $n^{\circ}$ 6, ch. 6: Rupture discontinue, rupture et glissement, p. 85.

HUDSON, J.A.; BROWN, E.T.; FAIRHURST, C. (1971). - Shape of the complete stress-strain curve for rock. - 13th Symp. on Rock Mechanics, sept. 71, pp. 773-795.

MANDEL, J. (1966). - Mécanique des milieux continus. - Gauthier-Villars, Paris, Déformation plastique hétérogène, tome II, p. 708.

MEYERHOFF, G.G. (1961). - Fondations superficielles: discussion, C.R. $5^{e}$ Cong. Int. mécanique des sols (Paris), tome III, p. 193.

ROSCOE, K.H. (1970). - The influence of strains in soil mechanics. - Géotechnique, vol. XX, 2, june 1970, pp. $129-170$.

SUEMINE, A. (1983). - Observational study on landside mechanism in the area of cristalline schist (Part I), An example of propagation of Ranikine state. - Bull. of the Disaster Prevention Inst., sept. 83, vol. 3, part. 3, pp. 105-127. 
